\title{
La globalización de la rendición de cuentas en el ámbito educativo: una revisión de factores y actores de difusión de políticas
}

\author{
A globalização da prestação de contas no âmbito educativo: uma revisão de \\ fatores e atores de difusão de políticas \\ The globalization of accountability in educational context: a review of political \\ diffusion factors and actors
}

ANTONI VERGER

LLUÍS PARCERISA

Resumo: $\mathrm{O}$ artigo analisa os fatores e condições que favoreceram a disseminação de políticas de prestação de contas a escala global e o papel desempenhado pelas organizações internacionais (OIs) al respeito. Metodologicamente, o artigo é baseado em uma scoping review que inclui 51 artigos. Os resultados mostram que a globalização da prestação de contas deve-se à confluência de uma grande variedade de fatores de natureza muto diferente, e identifica convergências e divergências entre os OIs na promoção da prestação de contas globalmente.

Palavras-chave: Prestação de contas; Governança regulatória; Probas estandarizas; Política educacional; Governança Global; Organizações internacionais; Nova Gestão Pública.

\begin{abstract}
The article analyses the factors and conditions that have favoured the diffusion of accountability policies in education worldwide, and the role played by international organizations (IO's) in this respect. Methodologically, the article is based on a scoping review that includes 51 articles. The article shows that the globalization of accountability is the result of the confluence of drivers and factors of a different nature, and reflects on both the converging and diverging ideas of the most relevant IOs in promoting accountability reforms globally.
\end{abstract}

Keywords: accountability; education policy; standardized tests; global governance; International Organizations; New Public Management.

Resumen: El artículo analiza los factores y condiciones que han favorecido la difusión de las políticas de rendición de cuentas a escala global, y el papel desempeñado por las organizaciones internacionales (OI) más prominentes en el campo educativo al respecto. Metodológicamente, el artículo se basa en una aproximación a la revisión de la literatura conocida como scoping review que incluye 51 artículos. El artículo muestra que la globalización de la rendición de cuentas 
en educación es el resultado de la confluencia de factores de una naturaleza muy diferente, e identifica las convergencias y divergencias entre diferentes OIs a la hora de promover reformas basadas en la rendición de cuentas.

Palabras clave: Rendición de Cuentas; pruebas estandarizadas; Política Educativa; Gobernanza Global; Organizaciones Internacionales; Nueva Gestión Pública.

\section{INTRODUCCIÓN}

En las últimas décadas, se han producido cambios profundos en la gobernanza de los servicios públicos. Actualmente, cada vez son más los gobiernos que optan por gestionar sus servicios de forma más indirecta: subcontratando a proveedores privados y otorgando más autonomía en determinados ámbitos de gestión a los proveedores públicos, descentralizando una parte de la gestión de los servicios en gobiernos locales, y/o incorporando agencias reguladoras y evaluadoras independientes en la administración pública (Scott 2000). Estos cambios en materia de gobernanza no implican necesariamente un debilitamiento de la autoridad del estado sobre los servicios públicos. Más bien, conllevan un giro en las funciones y el rol del estado en el ámbito de los servicios, y la necesidad de adoptar nuevas tecnologías con las que el estado pueda continuar ejerciendo control sobre éstos. En este contexto, junto a los mecanismos de financiación pública, las políticas de rendición de cuentas ( $\mathrm{RdC}$ ) han devenido un dispositivo fundamental para que la administración pública pueda controlar las prioridades y el desempeño de unos servicios públicos subministrados por proveedores cada vez más independientes (Lodge 2004).

La RdC es definida como "una relación entre un actor y un foro en la que el actor tiene la obligación de explicar y justificar su conducta, el foro puede plantear preguntas y emitir juicios, y el actor puede enfrentar consecuencias" (BOVENS, 2007, p.450). A pesar de que la RdC es un concepto polisémico y que puede asociarse a múltiples tipos de políticas, el modelo de $\mathrm{RdC}$ que se está extendiendo más intensamente en la actualidad tiene un carácter gerencial y centrado en resultados. Se trata de un enfoque de RdC que, en el caso del sector educativo, se centra en los resultados de aprendizaje de los estudiantes e implica la generación de datos a través de instrumentos de evaluación estandarizada (HAMILTON et al., 2002). A través de evaluaciones externas (y de la distribución de incentivos en función de los resultados obtenidos en dichas evaluaciones), el Estado puede delimitar las metas y los resultados que se esperan de las escuelas, y controlar de forma más efectiva el trabajo y el comportamiento del personal docente y el de otros agentes educativos. 
La RdC basada en pruebas externas representa una nueva fuente de presión para las escuelas y para los maestros. La RdC tiene por objetivo favorecer la transparencia de los servicios educativos, pero ello se traduce a menudo en el sometimiento del desempeño docente al mayor escrutinio de las autoridades educativas, de las familias e incluso de los medios de comunicación. En gran medida, el modelo de $\mathrm{RdC}$ que predomina actualmente desafía las dinámicas de autorregulación que habían prevalecido en la profesión docente, especialmente en aquellos países con estados de bienestar más avanzados, y refuerza la presencia y la capacidad reguladora de agentes externos a la propia profesión.

La RdC basada en pruebas externas ${ }^{1}$ es un fenómeno que ha adquirido un alcance global (SMITH, 2016). La literatura reciente muestra cómo dicho modelo de $\mathrm{RdC}$ ha penetrado en una amplia gama de sistemas educativos, incluyendo el Reino Unido y la Europa continental (GREK, et al., 2009; OZGA, 2013; VESELEY, 2012; Verger and Curran, 2014), los llamados países nórdicos (ELSTAD et al., 2009; MOOS, 2013; MOLLER; SKEDSMO, 2013), los Estados Unidos (LIPMAN, 2002, HURSH, 2005), Canadá (LEICHT et al., 2009), Australia y Nueva Zelanda (CODD, 2005; LINGARD, 2010) y Latinoamérica (BROOK, 2006; PARCERISA; FALABELLA, 2017).

La difusión internacional de la RdC como modelo de reforma educativa global es un fenómeno que llama la atención por, al menos, dos motivos. En primer lugar, porque países de diferentes regiones del mundo, con legados administrativos, sistemas educativos y niveles de desarrollo económico muy diferentes, parecen converger en la necesidad de adoptar políticas de RdC en el ámbito educativo. Y, en segundo lugar, porque cada vez más países están adoptando políticas de $\mathrm{RdC}$ a pesar de que la evidencia sobre los efectos positivos de estas políticas es todavía débil y, de hecho, poco concluyente. La investigación empírica sobre RdC y educación obtiene resultados muy diferentes - e incluso contradictorios - por lo que respecta a los efectos de las políticas de $\mathrm{RdC}$ sobre el incremento de los niveles de aprendizaje, la mejora de los procesos educativos, o la reducción de las desigualdades educativas. Además, a pesar de que los sistemas de RdC tienen por objetivo de promover la transparencia y la calidad en la educación, cada vez hay más estudios que muestran que, según cómo sean diseñados e implementados, los sistemas de $\mathrm{RdC}$ pueden generar resultados inesperados e incluso comportamientos no deseados en las escuelas (AU, 2007). En general, todavía no contamos con una base empírica lo suficientemente amplia para comprender las circunstancias y los mecanismos a través de los cuales la RdC podría lograr los resultados esperados.

$1 \quad$ A pesar de que existen diferentes modelos de $\mathrm{RdC}$ de cuentas, a partir de ahora, siempre que nos refiramos a la $\mathrm{RdC}$ en este artículo, nos referimos al modelo de $\mathrm{R} d C$ basado en pruebas estandarizadas externas. 
Frente a esta realidad, este artículo analiza las dinámicas a través de las cuales se ha difundido y adoptado la $\mathrm{RdC}$ como una herramienta fundamental en la gobernanza educativa a a escala global. El artículo se centra en las razones, factores y actores que favorecen la difusión internacional de la RdC. Concretamente, el artículo se estructura en dos partes principales. En la primera parte del artículo, exploramos aquellos factores que han favorecido de manera más determinante la difusión y la adopción de políticas de $\mathrm{RdC}$ en los sistemas educativos de muchos puntos del planeta, mientras que en la segunda parte nos centramos en el rol que han jugado las organizaciones internacionales más relevantes en el campo educativo a la hora de promocionar medidas de $\mathrm{RdC}$ en educación.

Metodológicamente, esta investigación se basa en el método de revisión de alcance (scoping review, en inglés), que tiene como objetivo identificar las principales tendencias y resultados en un campo de estudio específico. Este método de revisión de la literatura permite mapear la literatura existente sobre un tema determinado en un período relativamente breve de tiempo. Para el propósito de esta revisión en particular, hemos utilizado principalmente dos bases de datos científicas: Web of Science (WoS) y SCOPUS (véanse los términos de la búsqueda en el Apéndice 1). Adicionalmente, realizamos una búsqueda manual en libros y revistas clave, literatura gris y documentos elaborados por organizaciones internacionales. En total, identificamos un total de 51 documentos sobre la difusión internacional de las políticas de $\mathrm{RdC}$ en educación. Todos estos documentos fueron publicados entre los años 1995 y 2015.

\section{LA EMERGENCIA DE LA RENDICIÓN DE CUENTAS: FACTORES Y CONDICIONES}

Los factores que han llevado a la difusión global de la $\mathrm{RdC}$ en el ámbito educativo son numerosos. Como desarrollamos en esta sección, entre los principales factores se incluyen: avances técnicos y metodológicos en el campo de la evaluación educativa; motivaciones de naturaleza económica para la mejora de los resultados de aprendizaje; la expansión de ideas gerenciales basadas en la Nueva Gestión Pública (NGP); la difusión de políticas educativas globales que retro-alimentan la RdC; y la emergencia de una industria educativa cuya actividad gira alrededor de las pruebas de aprendizaje. 


\section{AVANCES METODOLÓGICOS Y TÉCNICOS EN LA MEDICIÓN DE LOS RESULTADOS DE APRENDIZAJE}

Los avances metodológicos y técnicos en la definición de los estándares de aprendizaje y en la medición de los resultados de aprendizaje, también han contribuido a la expansión de la $\mathrm{RdC}$ a escala mundial. Por razones obvias, la evolución metodológica en el campo de las pruebas de aprendizaje estandarizadas así como de los modelos de valor añadido (con los que medir el impacto de una determinada escuela o maestro en el desempeño de sus alumnos) es una condición necesaria para el desarrollo de sistemas de $\mathrm{RdC}$ basados en resultados más sofisticados y precisos.

El Programa Internacional para la Evaluación de Estudiantes (PISA) de la Organización para la Cooperación y el Desarrollo Económico (OCDE), y evaluaciones internacionales similares (como TIMSS o PIRLS), han contribuido a que los políticos y los tomadores de decisiones de políticas se sumen a una "carrera educativa" global para la consecución de mejores resultados de aprendizaje. En el contexto de esta carrera educativa internacional, la adopción de sistemas nacionales de evaluación estandarizados deviene una pieza fundamental y estratégica de competitividad. Sin embargo, las evaluaciones internacionales a gran escala como PISA no sólo ha contribuido a la introducción de presión competitiva en diferentes países para la obtención de mejores resultados, sino que también se ha convertido en un instrumento para transferir la tecnología con la que evaluar los resultados de aprendizaje a nivel nacional. En este sentido, no son pocos los países que han emulado la lógica y el contenido de las pruebas PISA en sus sistemas de evaluación nacionales (MEYER; BENAVOT, 2013; LINGARD et al, 2016).

La transferencia de tecnologías de evaluación estandarizada a nivel nacional se ha visto reforzada por el hecho de que la medición de los resultados del aprendizaje se ha convertido en el proxy más comúnmente aceptado para determinar la "calidad" de la educación. Los "resultados de aprendizaje" se han erigido en el indicador internacional de calidad educativa por excelencia debido a que se trata de un indicador concreto, conmensurable y fácilmente comparable. $\mathrm{Al}$ menos, se trata de un indicador que ofrece todo tipo de ventajas en relación a otros componentes de la calidad educativa que son más sensibles al contexto o más difíciles de capturar mediante pruebas de evaluación estandarizadas, como por ejemplo, las variables relacionadas con procesos educativos, la pedagogía, o la formación y desarrollo profesional docente (SAYED, 2011). No obstante, la centralidad actual de los resultados de aprendizaje como meta de excelencia 
educativa no responde únicamente a razones metodológicas sino que, como desarrollamos en los puntos siguientes, a motivaciones de carácter político y económico.

\section{PRESIONES ECONÓMICAS PARA LA MEJORA DE LOS RESULTADOS DE APRENDIZAJE}

En una economía global, cada vez más gobiernos y agentes económicos y sociales perciben la adquisición de aptitudes y competencias alineadas con las nuevas demandas del mercado laboral como un elemento clave a la hora de reforzar su competitividad económica (CARNOY; RHOTEN, 2002). Muchos de estos agentes se hacen eco además de nuevas fuentes de evidencia que conciben los "resultados de aprendizaje" como uno de los factores clave a la hora de explicar el crecimiento económico de los países (véase HANUSHEK et al., 2003). Como consecuencia de todo ello, la mayoría de reformas educativas actuales se centran en promover mejoras en el desempeño del alumnado, por lo que la medición de los resultados de aprendizaje deviene una condición necesaria para valorar el grado de éxito de dichas reformas. En general, los resultados de aprendizaje se han erigido en un punto de referencia fundamental a la hora de evaluar hasta qué punto "funcionan" o no las políticas educativas, así como la calidad de la educación (véase el punto anterior). En este contexto, las pruebas externas estandarizadas se han convertido en un componente central de un enfoque de reforma educativa que sitúa y define a la escuela "como el lugar para aumentar la competitividad económica del estado” (FOSTER, 2004, citado en KOYAMA, 2013, p.82).

La ley federal No Child Left Behind (NCLB), promulgada por el presidente de los Estados Unidos George W. Bush Jr a principios del siglo XXI, es probablemente el ejemplo más conocido y paradigmático de este enfoque de reforma educativa. La ley NCLB promueve una reforma de RdC centrada en la evaluación del rendimiento de los estudiantes, principalmente en relación a dos materias clave: matemáticas y lengua. NCLB establece los objetivos de aprendizaje que deben lograr todos los estudiantes, así como un sistema de incentivos para las escuelas vinculado a los resultados obtenidos por los estudiantes en las pruebas externas. Algunos de estos incentivos se dan en forma de sanciones. Por ejemplo, NCLB fomenta que los padres de escuelas con malos resultados en las pruebas puedan cambiar sus hijos a una escuela mejor (STECHER; HAMILTON; GONZÁLEZ, 2003). Además, después de cinco años de no cumplir con los objetivos de desempeño establecidos, NCLB contempla la posibilidad de que el estado cierre la escuela, o la convierta en una escuela charter. 


\section{NUEVA GESTIÓN PÚBLICA Y REFORMAS DEL SECTOR PÚBLICO}

El surgimiento y la consolidación de la Nueva Gestión Pública (NGP), como paradigma de reforma del sector público, pone énfasis en que los servicios públicos sean administrados de manera más independiente (es decir, a través de unidades gerenciales más pequeñas) y según el logro de resultados medibles (GUNTER et al., 2016; SCOT'T, 2000). En general, la NGP puede definirse como "un enfoque de la administración pública que emplea los conocimientos y las experiencias adquiridas en la gestión empresarial y otras disciplinas para mejorar la eficiencia, la eficacia y el desempeño general de los servicios públicos en las burocracias modernas" (VIGODA, 2003, p. 813). La NGP, en el ámbito educativo, se traduce en la introducción de nuevas formas de liderazgo educativo de corte gerencialista, y en la fragmentación del sistema educativo en unidades escolares más autónomas y diversas cuyo desempeño debe ser evaluado de forma regular por el estado a través de agencias de evaluación educativa (MOLLER; SKEDSMO, 2013).

Durante la década de 1980, el paradigma de la NGP fue adoptado por gobiernos conservadores y de la nueva derecha, que tenían como objetivo principal promover servicios públicos que fueran más eficientes y rentables (véase Tolofari, 2005). Sin embargo, hoy en día, la NGP es concebida como un enfoque de reforma válido por un mayor abanico de ideologías políticas. Actualmente, las medidas de NGP forman parte también de la agenda reformista de los gobiernos socialdemócratas (VERGER; NORMAND, 2015). Los partidos socialdemócratas, bajo la influencia de la llamada "tercera vía" que emergió durante los años noventa, comenzaron a adoptar medidas de NGP en el ámbito educativo -incluyendo la autonomía escolar y la rendición de cuentas- como una forma de promover no sólo la eficiencia económica sino que también la equidad y la diversificación de la servicios educativos.

Más allá del rol de las ideologías políticas, cabe tener en cuenta que la adopción de políticas de $\mathrm{RdC}$ representa un tipo de reforma educativa atractivo para muchos gobernantes ya que, por regla general, resulta políticamente rentable (POLLIT; BOUCKAERT, 2011). La implementación de sistemas de RdC permite a los gobiernos transmitir a los votantes -y a la ciudadanía en general- el mensaje de que están trabajando arduamente para el cambio educativo, y para la mejora de los resultados de aprendizaje y de las oportunidades futuras de los niños y niñas. $\mathrm{Al}$ mismo tiempo, las reformas de $\mathrm{RdC}$ tienden a implicar un bajo riesgo político ya que, en gran medida, la presión de la reforma recae en las escuelas y en los 
maestros, y no tanto en el gobierno. Cabe tener en cuenta además que este tipo de reformas acostumbran a ser "más baratas y rápidas que reformas alternativas" (SMITH et al., 2004, p.50).

\section{ALINEACIÓN CON OTRAS POLÍTICAS EDUCATIVAS GLOBALES}

La difusión internacional de políticas de autonomía escolar y las reformas curriculares basadas en estándares también han generado un contexto propicio para la adopción de sistemas de RdC. En el modelo predominante de reforma educativa actual, la autonomía escolar y la RdC tienden a ser concebidas como dos elementos indisociables y que se retro-alimentan. Ello se debe a que los gobiernos están dispuestos a conceder más autonomía a las escuelas (en términos organizativos, presupuestarios y/o pedagógicos), siempre y cuando las escuelas acepten someterse a procesos de supervisión y evaluación más estrictos. Como señala la OCDE (2013, p. 45), “a mayores responsabilidades asumidas por las escuelas, mayores requisitos de $\mathrm{RdC}$ como la evaluación externa de las escuelas y la publicación de información sobre el rendimiento de los estudiantes".

La RdC también se ve reforzada por el movimiento de reforma basado en estándares (standards-based reform movement). El establecimiento de estándares básicos comunes en las reformas curriculares conduce a un énfasis creciente en las pruebas externas, las recompensas y las sanciones (Darling-Hammond, 2004). En cierto modo, los conocidos como common core standards que muchos países adoptan en el ámbito curricular definen cuáles deberían ser los resultados de aprendizaje del alumnado, por lo que las evaluaciones externas devienen la principal herramienta en manos de los gobiernos para averiguar si las escuelas cumplen con dichas expectativas de aprendizaje.

\section{LA EMERENCIA DE UNA INDÚSTRIA DE PRUEBAS DE APRENDIZAJE A NIVEL GLOBAL}

Por último, también cabe poner de relieve que, en paralelo a la emergencia de reformas de $\mathrm{RdC}$, se ha desarrollado una industria de pruebas de evaluación ("testing industry"). Este sector de la industria educativa está muy interesado en la expansión y profundización de las reformas de $\mathrm{RdC}$ y de la adopción de sistemas de evaluación del desempeño educativo. La industria de las pruebas de aprendizaje se ha convertido en uno de los sectores más lucrativos en el contexto de la denominada industria educativa global (VERGER; LUBIENSKI; STEINER- 
KHAMSI, 2016). Empresas como Pearson se han especializado en la evaluación y el seguimiento de los resultados de aprendizaje de los niños y niñas y, sobre la base de estos datos, venden servicios de mejoramiento de la educación a los países, gobiernos locales, escuelas y/o familias (HOGAN et al., 2016). Otras empresas de evaluación educativa obtienen importantes contratos para la implementación y/o el procesamiento de los datos de pruebas externas tanto nacionales como internacionales (ADDEY; SELLAR 2018).

Por todas estas razones, las empresas que proveen servicios relacionados con la medición de resultados de aprendizaje también son agentes activos en la propagación de reformas de rendición de cuentas. Según la OCDE, el hecho de que "la evaluación estandarizada de los estudiantes se convierta en una industria más rentable" significa que "las empresas tienen fuertes incentivos para presionar por la expansión de la evaluación estandarizada de los estudiantes como una política educativa que influye en las actividades dentro del marco de evaluación" (OCDE, 2013 , p.51). En una línea de razonamiento similar, Carnoy (2016, p.36) considera que "los fabricantes de pruebas tienen un interés económico particular en que los sistemas educativos y las escuelas cambien su concepción de conocimiento académico o incluso de conocimiento útil para ajustarse así a la prueba particular que venden".

\section{EL PAPEL DE LAS ORGANIZACIONES INTERNACIONALES EN LA DIFUSIÓN DE LA RENDICIÓN DE CUENTAS}

La influencia ejercida por las organizaciones internacionales más prominentes en el campo educativo también explica la expansión internacional de las reformas de RdC. Muchas organizaciones internacionales tienen la capacidad no sólo de financiar la implementación de reformas de $\mathrm{RdC}$, sino que también de promover dinámicas de emulación entre sus países miembros, y de favorecer así la creación de un consenso alrededor de la deseabilidad de adoptar sistemas de $\mathrm{RdC}$ en el campo educativo. Como desarrollamos a continuación, organizaciones internacionales como la OCDE, el Banco Mundial y, más recientemente, la Organización de las Naciones Unidas para la Educación, la Ciencia y la Cultura (UNESCO) han contribuido a promover las medidas de RdC como una solución clave a la hora de abordar muchos de los problemas que enfrentan los sistemas educativos contemporáneos. 


\section{LA OCDE}

La autonomía escolar con medidas de $\mathrm{RdC}$ es una de las recomendaciones de políticas que aparecen de forma más reiterada en los informes PISA de la OCDE, así como en otros informes de esta organización internacional (véase OCDE, 2011). Muchos de los estudios de caso revisados para la elaboración de este artículo coinciden en señalar a la OCDE, y al informe PISA en particular, como impulsores clave de las reformas de RdC a nivel nacional. De hecho, según un estudio reciente, 29 representantes de países de la OCDE (de un total de 37) admitieron que las recomendaciones de PISA/OCDE sobre la RdC han influido de forma significativa en procesos de formulación de políticas a nivel nacional (BREAKSPEAR, 2012). De manera quizás más indirecta, el shock que algunos países han experimentado a raíz de sus malos resultados en PISA -especialmente después de las primeras ediciones de esta evaluación internacional- también ha promovido la introducción de reformas de $\mathrm{RdC}$ a nivel nacional (ELSTAD et al., 2009).

La División de Educación de la OCDE aboga principalmente por formas de $\mathrm{RdC}$ gerenciales y basadas en pruebas de aprendizaje externas. Sin embargo, esta organización también hace hincapié en que los sistemas de RdC deben ser holísticos y centrarse en mejorar las prácticas educativas en las aulas, y no poner excesivo énfasis en los resultados de aprendizaje (véase OCDE, 2012, 2013). La OCDE no está en contra de la publicación de los resultados de las escuelas, pero afirma que los resultados deben hacerse públicos de una manera "justa" y "razonable". Al mismo tiempo, la OCDE también considera que es necesario "alinear la evaluación externa de las escuelas" con políticas internas "autoevaluación" (OCDE, 2013, p.8). La OCDE también destaca que la adopción de este tipo de políticas debe ser acordada con los principales agentes educativos:

To be designed successfully, evaluation and assessment frameworks should draw on informed policy diagnosis and best practice, which may require the use of pilots and experimentation. To be implemented successfully, a substantial effort should be made to build consensus among all stakeholders, who are more likely to accept change if they understand its rationale and potential usefulness (OECD, 2013, p.14).

La OCDE, sobre la base de los datos del PISA, considera la RdC y la autonomía escolar son dos políticas que se retro-alimentan y se refuerzan positivamente. Según la OCDE/PISA, las escuelas con mayor autonomía en la asignación de recursos muestran mejores resultados de los estudiantes en el contexto de regímenes de $\mathrm{RdC}$ fuertes (es decir, en sistemas de $\mathrm{RdC}$ en los cuales los gobiernos hacen públicos los resultados del desempeño de las escuelas). Ahora

672 - RBPAE - v. 33, n. 3, p. 663 - 684, set./dez. 2017 
bien, la OCDE también matiza que la relación opuesta también es cierta, ya que "en los países donde no existen tales mecanismos de rendición de cuentas, las escuelas con mayor autonomía en la asignación de recursos tienden a empeorar" (OCDE, 2011, p.1), de manera que países con sistemas educativos con poca autonomía de centro y RdC débil podrían obtener también buenos resultados.

\section{EL BANCO MUNDIAL}

El Banco Mundial aboga por modelos de RdC que promueven dinámicas de mercado y elección escolar, así como por medidas de RdC que empoderen a las familias y a la comunidad ante la escuela (EDWARDS, 2012). El Banco Mundial difunde activamente estas formas de $\mathrm{RdC}$ a través de sus numerosos informes, operaciones de préstamo y, más recientemente, a través del Enfoque de Sistemas para Mejores Resultados Educacionales (SABER). Según el marco de SABER sobre la autonomía escolar y la $\mathrm{RdC}$, "aumentar la accountability de las escuelas es una condición necesaria para mejorar la calidad de los docentes" (WORLD BANK, 2015, p.4). Y añade que la RdC "mejora el aprendizaje porque [esta política] alinea los incentivos de los docentesy las familias" (WORLD BANK, 2015, p.2). El Banco Mundial también se hace eco del mensaje de la OCDE sobre la importancia de fragmentar el sistema educativo en escuelas más independientes y autónomas, al mismo tiempo que se promueven medidas más estrictas de RdC:

\footnotetext{
School autonomy must be complemented with school accountability to promote academic excellence. This has been well documented through various impact evaluations. We also know that the highest PISA scores come from countries where autonomy and accountability are implemented together (OECD 2011) (WORLD BANK, 2015, p.36)
}

Para el Banco Mundial, la RdC es una pieza clave en el enfoque sistémico de reforma educativa que promueve en el marco de la Estrategia de Educación 2020 "Aprendizaje para todos". Este documento, que define la política educativa del Banco Mundial en el período 2010-2020, considera que "la mejora del desempeño y de los resultados depende de un cuidadoso equilibrio entre tres instrumentos de política que influyen en el comportamiento de los actores locales: 1) mayor autonomía a nivel local; (2) reforzar las relaciones de rendición de cuentas; y (3) sistemas de evaluación eficaces" (WORLD BANK, 2011, p.33).

Esta organización internacional considera que la "vía corta de la rendición de cuentas" (que opera a través de la elección escolar y el school-based management) tiene ventajas sobre la "vía larga de rendición de cuentas" (es decir, sobre modelos de rendición de cuentas más burocráticos y que dependen 
de la mediación del estado), ya que "la vía corta ofrece a los clientes el poder de proporcionar comentarios y feedback con mayor frecuencia a los proveedores educativos para hacerles saber cómo se están desempeñando y para hacerlos responsables de garantizar unos servicios de buena calidad" (WORLD BANK, 2015, p.5). En general, para esta organización internacional, las formas postburocráticas de $\mathrm{RdC}$ como el school-based management no sólo son inherentemente deseables, sino la solución más apropiada en el contexto de los países de bajos ingresos, ya que estos países no cuentan con los recursos ni con la capacidad administrativa necesaria para poner en marcha otros sistemas de RdC (BRUNS et al., 2011, WORLD BANK, 2006).

\section{LA UNESCO}

El Objetivo de Desarrollo Sostenible número 4 (ODS-4), que es el Objetivo de Desarrollo Sostenible de las Naciones Unidas centrado en la educación, hace especial hincapié en promover la mejora de los resultados del aprendizaje. De las siete metas incluidas en el ODS-4, cinco se centran en los resultados de aprendizaje y la adquisición de competencias específicas (en lectura, matemáticas, ciudadanía global, cultura de paz, etc.). El giro del enfoque del acceso escolar al aprendizaje es probablemente el cambio más significativo que se puede observar entre, por un lado, las agendas de los Objetivos de Desarrollo del Milenio y la Educación para Todos aprobadas en el año 2000 y, por el otro, la agenda actual de los ODS aprobada en el año 2015. El documento Educación 2030 - que es el marco de acción acordado por la comunidad internacional, bajo la dirección de la UNESCO, para avanzar en el ODS 4 -, promueve sistemas de rendición de cuentas que se basan, al menos parcialmente, en la medición de resultados de aprendizaje.

La rendición de cuentas es uno de los conceptos más utilizados en el marco de acción Educación 2030. Este marco concibe la creación de sistemas nacionales de rendición de cuentas comprehensivos (es decir, centrados en los resultados del aprendizaje, pero también en otras dimensiones) como un aspecto indispensable en el monitoreo del alcance de las nuevas metas educativas. Según la UNESCO, "el control de la calidad en la educación requiere un enfoque multidimensional, que abarque el diseño del sistema, los insumos, el contenido, los procesos y los resultados". (UNESCO 2015: 17)

A diferencia del Banco Mundial, la UNESCO no promueve formas de rendición de cuentas de mercado ni de alto riesgo (lo que en el mundo anglosajón se conoce como high-stakes accountability). De hecho, esta organización internacional es generalmente escéptica con respecto a las asunciones sobre los beneficios de 
los mecanismos de mercado en educación y/o de la aplicación de sanciones para escuelas y docentes (UNESCO 2009). En el Informe de Seguimiento de la Educación en el Mundo del año 2017, dedicado al tema de rendición de cuentas en educación, la UNESCO se muestra favorable a la RdC como un principio rector de los sistemas educativos, pero también adopta un enfoque cauteloso al enfatizar los posibles efectos no deseados de los sistemas de rendición de cuentas. Algunos de sus mensajes clave en este informe son que:

- "Si se hace responsables [a los maestros o a las escuelas] de resultados que escapan a su control, éstos tratarán de evitar riesgos, minimizar su rol o ajustar su comportamiento de forma involuntaria para protegerse.

- La confianza se ausenta del sistema educativo cuando los actores [escolares] operan con miedo al castigo. Un propósito compartido, que fomenta la confianza, es fundamental para la rendición de cuentas efectiva. "(UNESCO, 2017, np)

La UNESCO propone un modelo de RdC mutua o compartida, es decir, un modelo de rendición de cuentas en el que los gobiernos, y no solo las escuelas y los docentes, sean los que tengan que rendir cuentas. También apuesta por un modelo de $\mathrm{RdC}$ de carácter participativo. Dada la naturaleza contenciosa de la RdC en el campo de la política educativa, la UNESCO considera importante diseñar sistemas de $\mathrm{RdC}$ de forma consensuada, inclusiva y participativa:

\footnotetext{
As the primary responsibility for monitoring lies at the country level, countries should build up effective monitoring and accountability mechanisms, adapted to national priorities, in consultation with civil society. This includes building greater consensus as to what specific quality standards and learning outcomes should be achieved across the life course - from early childhood development to adult skills acquisition and how they should be measured (UNESCO 2015, p.17)
}

Como hemos podido observar, las principales organizaciones internacionales en el campo de la política educativa convergen a la hora de promover formas de $\mathrm{RdC}$ basadas en pruebas externas como una forma de garantizar estándares mínimos de aprendizaje en diferentes países. Sin embargo, el Banco Mundial alinea sus políticas de RdC con la promoción de mecanismos de mercado y la gestión comunitaria de las escuelas, mientras que la OCDE está más inclinada a combinar la $\mathrm{RdC}$ con evaluaciones de carácter formativo y sistemas de autoevaluación de las escuelas. La UNESCO, por su parte, aboga por sistemas de $\mathrm{RdC}$ comprehensivos que operen a múltiples niveles y en múltiples direcciones de manera que la $\mathrm{RdC}$ no conciba, al menos de manera exclusiva, a los profesores y a las escuelas como los principales responsables de la calidad educativa. 


\section{CONCLUSIONES}

En gran medida, el éxito de la $\mathrm{RdC}$ recae en que se la concibe como una solución política efectiva y pragmática ante la amplia gama de problemas complejos que afrontan la mayoría de sistemas educativos del planeta. Nos referimos a problemas tan diversos como la eficiencia (la RdC puede contribuir a alinear las aspiraciones gubernamentales en materia educativa con los objetivos que las escuelas tratan de alcanzar), la eficacia ( $\mathrm{RdC}$ como herramienta para la mejora del desempeño educativo de las escuelas, los docentes y los estudiantes), la equidad (la $\mathrm{RdC}$ como un instrumento con el que garantizar que todos los estudiantes alcancen un nivel mínimo de competencias básicas en determinadas materias) y la buena gobernanza (la $\mathrm{RdC}$ como un mecanismo que puede promover la transparencia y el control democrático en los servicios educativos). No obstante, en este artículo, hemos visto que la expansión y el alcance global de las políticas de $\mathrm{RdC}$ en educación se explican también por factores que trascienden el ámbito educativo. Como hemos visto, una amplia gama de factores de naturaleza muy diversa (económica, tecnológica, política, discursiva, etc.) están contribuyendo a la intensa difusión de la $\mathrm{RdC}$ en el campo educativo, y a su consolidación como un elemento central en la regulación de los sistemas educativos en muchos puntos del planeta.

Un factor que explica el éxito de la RdC es el de su calidad como "recipiente vacío", es decir, un concepto maleable al que se puede otorgar significados muy variados (STEINER-KHAMSI, 2016). A raíz de su carácter polisémico, la RdC permite alinear las preferencias políticas de una amplia variedad de actores, entre los que encontramos organizaciones internacionales, gobiernos y el sector privado, entorno a un programa aparentemente similar de reforma educativa. Las organizaciones internacionales más relevantes en el campo de la política educativa también han contribuido a generar altas expectativas respecto a las reformas de $\mathrm{RdC}$ en educación. Sin embargo, a pesar de que estas organizaciones coinciden a la hora de favorecer la adopción de sistemas nacionales de RdC, divergen por lo que respecta a los objetivos e instrumentos concretos en los que se concretan los sistemas de RdC que promueven.

Además, la difusión internacional de la $\mathrm{RdC}$ no conlleva necesariamente que se estén difundiendo modelos homogéneos de RdC sobre el terreno (MARTINSSON, 2011). Como pone de relieve Van Zanten (2002, p. 302), "los estados no pueden evitar presiones globales de cambio que apuntan a direcciones específicas, pero pueden torcer y transformar [estas presiones] para adaptarlas a propósitos y oportunidades nacionales". Así, sobre el terreno, observamos que los sistemas de RdC basados en resultados de aprendizaje pueden diferir 
considerablemente: pueden estar vinculados a evaluaciones de alto riesgo ( $h i g h-$ stakes) o de bajo riesgo (low-stakes), ser más o menos comprehensivos en términos de las áreas de conocimiento que cubren, combinarse (o no) con diferentes formas de evaluación (pr ejemplo, servicios de inspección, auto-evaluación, etc.), complementarse con diferentes formas de apoyo del estado para la mejora educativa o, por el contrario, complementarse con mecanismos de mercado. Finalmente, incluso cuando pareciera que los sistemas de RdC adoptan formas muy similares, pueden traducirse en prácticas muy diferentes de acuerdo a cómo los actores educativos locales interpretan y ponen en práctica dichos sistemas a nivel de distrito, escuela y/o aula. En definitiva, este artículo constata que es necesario continuar desarrollando investigaciones empíricas con las que, por un lado, capturar la multiplicidad de trayectorias y traducciones de la $\mathrm{RdC}$ a nivel nacional y local y, por el otro, generar evidencias más robustas que nos permitan entender cómo y por qué distintos diseños de $\mathrm{RdC}$ conducen a comportamientos y a efectos aparentemente tan dispares en el ámbito de los sistemas educativos.

\section{BIBLIOGRAFÍA}

ADDEY, Camilla. \& SELLAR, Sam. Why do countries participate in PISA? Understanding the role of international large-scale assessments in global education policy. In Verger, A., Novelli, M. \& Altinyelken, H. K. (Eds.), Global Education Policy and International Development: New Agendas, Issues and Policies. New York: Bloomsbury Academic, 2018, Forthcoming.

ANDERSEN, Simon Calmar. The impact of public management reforms on student performance in Danish schools. Public Administration, v. 86, n. 2, p.541-558, 2008. https://doi.org/10.1111/j.1467-9299.2008.00717.x

AU, Wayne. High-stakes testing and curricular control: A qualitative metasynthesis. Educational Researcher, v.36, n. 5, p.258-267, 2007. https:// doi.org/10.3102/0013189X07306523

BARROSO, Joao. utilização do conhecimento em política: o caso da gestão escolar em Portugal, Educaçao \& Sociedade, v. 30, n. 109, p.987-1007. 2009. https://doi.org/10.1590/S0101-73302009000400004

BOVENS, Mark. Analysing and assessing accountability: a conceptual framework.

European law journal, v.13, n.4, p.447-468, 2007. https://doi.org/10.1111/ j.1468-0386.2007.00378.x 
BREAKSPEAR, Simon. The Policy Impact of PISA: An Exploration of the Normative Effects of International Benchmarking in School System Performance. OECD Education Working Papers, n.71. OECD Publishing, 2007. DOI: http://dx.doi.org/10.1787/5k9fdfqffr28-en

BROOKE, Nigel. The future of educational accountability policies in Brazil. Cadernos de Pesquisa, v. 36, n. 128, p. 377-401, 2006. https://doi.org/10.1590/ S0100-15742006000200006

BRUNS, Barbara, FILMER, Deon, and PATRINOS, Harry, A. Making Schools Work: New Evidence on Accountability Reforms. Washington, DC: World Bank Group, 2011. https://doi.org/10.1596/978-0-8213-8679-8

CARNOY, Martin; \& RHOTEN, Diana. What does globalization mean for educational change? A comparative approach. Comparative education review, v. 46, n. 1, p.1-9, 2002. https://doi.org/10.1086/324053

CARNOY, Martin. Educational policies in the face of globalization: Whither the Nation State? In: K. Mundy, K; Green, A; Lingard, R; \& Verger, A. (Eds.), Handbook of global policy and policy-making in education. West Sussex, UK: Wiley-Blackwell, 2016, p. 22-42. https://doi.org/10.1002/9781118468005. ch1

CODD, John. Teachers as 'managed professionals' in the global education industry: The New Zealand experience. Educational review, v. 57, n.2, p. 193206, 2005. https://doi.org/10.1080/0013191042000308369

DARLING-HAMMOND, Linda. Standards, accountability, and school reform. The Teachers College Record, v. 106, n. 6, p.1047-1085, 2004. https://doi. org/10.1111/j.1467-9620.2004.00372.x

EDWARDS, D. Brent. The approach of the World Bank to participation in development and education governance: Trajectories, frameworks, results. En Education Strategy in the Developing World: Revising the World Bank's Education Policy. Emerald Group Publishing Limited, 2012. p. 249-273.

ELSTAD, Eyvind; NORTVEDT, Guri. A; TURMO, Are. The norwegian assessment system: An accountability perspective. Cadmo, v. 17, n. 2, p. 89-103, 2009. 
EYBEN, Rosalind. Power, Mutual Accountability and Responsibility in the Practice of International Aid: A Relational Approach. IDS Working Paper 305, 2008.

GREK, Sotiria; LAWN, Martin; LINGARD, Bob; OZGA, Jenny; RINNE, Risto; SEGERHOLM, Christina; \& SIMOLA, Hannu. (2009). National policy brokering and the construction of the European Education Space in England, Sweden, Finland and Scotland. Comparative Education, v. 45, n. 1, p. 5-21, 2009. https://doi.org/10.1080/03050060802661378

HAMILTON, L. S., STECHER, B. M., \& KLEIN, S. P. (2002). Making sense of test-based accountability in education. Rand Corporation.

HANGARTNER, J., \& SVATON, C. J. (2013). From autonomy to quality management: NPM impacts on school governance in Switzerland. Journal of Educational Administration and History, Vol. 45, No. 4, pp. 354-369. https:/ / doi.org/10.1080/00220620.2013.822352

HANUSHEK, Erik; KAIN, John. F; MARKMAN, Jacob. M; \& RIVKIN, Steven. G. Does peer ability affect student achievement?. Journal of applied econometrics, v. 18, n. 5, p. 527-544, 2003. https://doi.org/10.1002/jae.741

HANUSHEK, Erik. A., \& WOESSMANN, Ludger. The role of cognitive skills in economic development. Journal of Economic Literature, v.46, n.3, p. $607-$ 668, 2008.

HOGAN, Anna; SELLAR, Sam; \& LINGARD, Bob. Corporate Social Responsibility and Neo-Social Accountability in Education: The Case of Pearson plc. In: Verger, A; LUBIENSKI, C; and STEINER-KHAMSI, G. (eds). World Yearbook of Education: The Global Education Industry. New York: Routledge. 2016, p. 107-124

HURSH, David. The growth of high-stakes testing in the USA: accountability, markets and the decline in educational equality. British Educational Research Journal, v.31,n.5, p.605-622,2005. https://doi.org/10.1080/01411920500240767 
KAMENS, David. H; \& BENAVOT, Aaron. National, regional and international learning assessments: Trends among developing countries, 1960-2009. Globalisation, societies and education, v. 9, n. 2, p. 285-300, 2011. https:// doi.org/10.1080/14767724.2011.577337

KOYAMA, Jill. Global scare tactics and the call for US schools to be held accountable. American Journal of Education, v. 120, n.1, p. 77-99, 2013. https://doi.org/10.1086/673122

LEICHT, Kevin, T; WALTER, Tony; SAINSAULIEU, Ivan; \& DAVIES, Scott. New Public Management and New Professionalism across Nations and Contexts, Current Sociology, v. 57, n. 4, p.581-605, 2009. https://doi. org/10.1177/0011392109104355

LINGARD, Bob. Policy borrowing, policy learning: Testing times in Australian schooling. Critical Studies in Education, v. 51, n. 2, p. 129-147, 2010. https:// doi.org/10.1080/17508481003731026

LINGARD, Bob; MARTINO, Wayne; REZAI-RASHTI, Goli; \& SELLA, Sam. Globalizing Educational Accountabilities. New York: Routledge, 2016.

LINN, Robert, L. Assessments and accountability. Educational Researcher, v. 29, n. 2, p. 4-16, 2000. https://doi.org/10.3102/0013189X029002004

LIPMAN, Pauline. Making the global city, making inequality: The political economy and cultural politics of Chicago school policy. American Educational Research Journal, v. 39, n. 2, p.379-419, 2002. https://doi.org/10.3102/00028312039002379

LODGE, Martin. Accountability and transparency in regulation: critiques, doctrines and instruments. In: Jordana, J; \& Levi-Faur, D. (eds). Politics of Regulation. CRC Series on Competition, Regulation and Development. Cheltenham: Edward Elgar Publishing, , 2004, p. 124-144.

MARTINSSON, Johanna. Global Norms: Creation, Diffusion, and Limits. Washington, DC: World Bank/Communication for Governance and Accountability, 2011.http://siteresources.worldbank.org/EXTGOVACC/ Resources/FinalGlobalNormsv1.Pdf, accessed, December 15, 2016. https:/ / doi. org/10.1596/26891 
MEYER, Heinz. Dieter; \& Benavot, Aaron. (Eds.). PISA, power, and policy: The emergence of global educational governance. Oxford: Symposium Books, 2013. https://doi.org/10.15730/books.85

MOLLER, Jorunn; SKEDSMO, Guri. Modernising education: New Public Management reform in the Norwegian education system. Journal of Educational Administration and History, Vol. 45, No. 4, pp. 336-353, 2013. https://doi.org /10.1080/00220620.2013.822353

MOOS, Lejf. School leadership in a contradictory world. Revista de Investigacion Educativa, Vol. 31, No. 1, pp. 15-29, 2013. https://doi. org/10.6018/rie.31.1.162511

OECD. School autonomy and accountability: Are they related to student performance? PIS A in Focus, No, 9, Paris: OECD Publishing, 2011.

OECD. Synergies for Better Learning: An International Perspective on Evaluation and Assessment. Paris: OECD Publishing, 2013.

OZGA, Jenny. Accountability as a policy technology: Accounting for education performance in Europe. International Review of Administrative Sciences, v. 79, n. 2, p. 292-309, 2013. https://doi.org/10.1177/0020852313477763

PALMER, Deborah; \& RANGEL, Virginia, Snodgrass. High Stakes Accountability and Policy Implementation: Teacher Decision Making in Bilingual Classrooms in Texas. Educational Policy, v. 25, n. 4, p. 614-647, 2011. https:// doi.org/10.1177/0895904810374848

PARCERISA, Lluís; \& FALABELLA, Alejandra. La Consolidación del Estado Evaluador a Través de Políticas de Rendición de Cuentas: Trayectoria, Producción y Tensiones en el Sistema Educativo Chileno. Education Policy Analysis Archives, v. 25, n.89, p. 1-27, 2017.

POLLIT'T, Christopher; \& BOUCKAERT, Geert. Public Management Reform: A comparative analysis-new public management, governance, and the Neo-Weberian state. Oxford: Oxford University Press, 2011.

SAYED, Yusuf. After 2015: time for an education quality goal?. Compare, v. 41, n. 1, p.129-130, 2011. https://doi.org/10.1080/03057925.2011.534847 
SCOT'T, Colin. Accountability in the regulatory state. Journal of law and society, v. 27, n. 1, p. 38-60, 2000.

SMITH, Mary Lee; MILLER-KAHN, LINDA; HEINECKE, Walter; \& JARVIS, PATRICIA F. Political Spectacle and the Fate of American Schools. New York: Routledge, 2004. https://doi.org/10.4324/9780203465554

SMITH, W. C. (Ed.). The global testing culture: Shaping education policy, perceptions, and practice. London: Symposium Books Ltd, 2016.

STECHER, Brian; HAMILTON, Laura; \& GONZALEZ, Gabriella. Working smarter to leave no child left behind. Santa Monica, CA: Rand Corporation, 2003.

STEINER-KHAMSI, Gita. New directions in policy borrowing research. Asia Pacific Education Review, v. 17, n. 3, p. 381-390, 2016.

TOLOFARI, Sowaribi. New public management and education. Policy Futures in Education, v. 3, n. 1, p. 75-89, 2005. https://doi.org/10.2304/pfie.2005.3.1.11

UNESCO. EFA Global Monitoring Report 2009. Overcoming inequality: why governance matters. Paris: UNESCO, 2009.

UNESCO. Framework for Action Education 2030: Towards inclusive and equitable quality education and lifelong learning for all, 2015. Available at: http://www.uis.unesco.org/Education/Documents/wef-framework-for-action. pdf

UNESCO, Global Education Monitoring Report 2017, Accountability in Education: Meeting our Commitments, 2017. Summary Version in http:// gem-report-2017.unesco.org/en/chapter/introduction-accountability/

VAN ZANTEN, Agnes. Educational change and new cleavages between head teachers, teachers and parents: global and local perspectives on the French case, Journal of Education Policy, v. 17, n. 3, p.289-304, 2002. https://doi. org/10.1080/02680930210127568 
VERGER, Antoni; \& CURRAN, Marta. New public management as a global education policy: its adoption and re-contextualization in a Southern European setting. Critical studies in education, v. 55, n. 3, p.253-271, 2014. https://doi.or g/10.1080/17508487.2014.913531

VERGER, Antoni; \& NORMAND, Romuald. New public management and education: theoretical and conceptual elements for the study of a global education reform model. Educação \& Sociedade, v. 36, n. 132, p. 599-622, 2015. https:// doi.org/10.1590/ES0101-73302015152799

VERGER, Antoni; LUBIENSKI, Christian; STEINER-KHAMSI, Gita. The emergence and structuring of the global education industry: Towards an analytical framework. In:

VERGER, A; LUBIENSKI, C; STEINER-KHAMSI, G. (eds.). The world yearbook of education 2016: The Global Education Industry. New York: Routledge, 2016.

VESELEY, Arnost. The Institutionalisation of Non-responsibility, Efficiency or Conformity? Organisational Reform of Public Services Based on Accountability Theory. Sociologicky Casopis-Czech Sociological Review, v. 48, n. 4, p.757784, 2012.

VIGODA, Eran. (2003). New public management. Encyclopedia of publicadministration and public policy, n. 2, p. 812-816.

WORLD BANK. Social Accountability Sourcebook. Washington DC: World Bank, 2006.

WORLD BANK. Learning for All: Investing in People's Knowledge and Skills to Promote Development. Washington DC: World Bank, 2011.

WORLD BANK. What Matters Most for School Autonomy and Accountability: A Framework Paper. SABER Working Paper Series, 9, Washington DC: World Bank, 2015. 


\section{APÉNDICE 1. PALABRAS CLAVE}

TITLE-ABS-KEY ("education"OR "education reform"OR "School") AND TITLE-ABS-KEY("International organi*ations"OR "OECD”OR "PISA"OR "PUMA"OR "world bank"OR "European Commission"OR "policy difussion"OR "policy adoption”OR “agenda-setting”OR "global education agenda"OR "policy entepreneur"OR "policy learning"OR "emulation"OR "dissemination"OR "globali*ation")AND TITLE-ABSKEY("New Public Management"OR "NPM"OR "School autonomy"OR "Institutional autonomy"OR "Accountability"OR "Merit-based pay" OR "School-based management"OR "Public management reform") AND NOT ("Higher education") AND (LIMIT-TO(SUBJAREA,"SOCI") OR LIMITTO(SUBJAREA,"BUSI") OR LIMIT-TO(SUBJAREA,"ECON") OR LIMITTO(SUBJAREA,"ARTS") ) AND (LIMIT-TO(SUBJAREA,"SOCI") OR LIMIT-TO(SUBJAREA,"BUSI") OR LIMIT-TO(SUBJAREA,"ECON" ) )

ANTONI VERGER é professor na Universidade Autônoma de Barcelona. E-mail: antoni.verger@uab.cat

LLUÍS PARCERISA é professor na Universidade Autônoma de Barcelona. E-mail: lluis.parcerisa@uab.cat

Recebido em dezembro de 2017 Aprovado em dezembro de 2017 